\title{
Primal/Dual Linear Programming and Statistical Atlases for Cartilage Segmentation ${ }^{\star}$
}

\author{
Ben Glocker ${ }^{1,2}$, Nikos Komodakis ${ }^{2,4}$, Nikos Paragios ${ }^{2}$, \\ Christian Glaser ${ }^{3}$, Georgios Tziritas ${ }^{4}$, and Nassir Navab ${ }^{1}$ \\ ${ }^{1}$ Computer Aided Medical Procedures (CAMP) \\ Technische Universität München \\ \{glocker, navab\}@in.tum.de \\ ${ }^{2}$ GALEN Group, Laboratoire de Mathématiques Appliquées aux Systèmes \\ Ecole Centrale de Paris \\ ${ }^{3}$ Department of Clinical Radiology, Ludwig-Maximilians-Universität München \\ ${ }^{4}$ Computer Science Department, University of Crete
}

\begin{abstract}
In this paper we propose a novel approach for automatic segmentation of cartilage using a statistical atlas and efficient primal/dual linear programming. To this end, a novel statistical atlas construction is considered from registered training examples. Segmentation is then solved through registration which aims at deforming the atlas such that the conditional posterior of the learned (atlas) density is maximized with respect to the image. Such a task is reformulated using a discrete set of deformations and segmentation becomes equivalent to finding the set of local deformations which optimally match the model to the image. We evaluate our method on 56 MRI data sets ( 28 used for the model and 28 used for evaluation) and obtain a fully automatic segmentation of patella cartilage volume with an overlap ratio of 0.84 with a sensitivity and specificity of $94.06 \%$ and $99.92 \%$, respectively.
\end{abstract}

\section{Introduction}

Degeneration of knee joint cartilage is an important and early indicator of osteoarthritis (OA) which is one of the major socio-economic burdens nowadays [1]. Accurate quantification of the articular cartilage degeneration in an early stage using MR images is a promising approach in diagnosis and therapy for this disease [2]. Particularly, volume and thickness measurement of cartilage tissue has been shown to deliver significant parameters in assessment of pathologies [3 4|5]. Here, accurate computer-aided diagnosis tools could improve the clinical routine where image segmentation plays a crucial role. In order to overcome the time-consuming and tedious work of manual segmentation, one tries to automate the segmentation as much as possible.

Many automatic and semi-automatic cartilage segmentation methods have been proposed. Folkesson et al. [6] propose a hierarchical classification scheme for automatic segmentation of cartilage in low field MR images. A semi-automatic method based on watershed transformation and pre-segmentation using [6] is presented by Dam et

\footnotetext{
* This work is partially supported by Siemens Medical Solutions, Erlangen, Germany.
} 
al. [7]. An earlier work by Grau et al. [8] proposes an extension to the standard watershed transformation for semi-automatic segmentation. Cheong et al. [9] compare different model-based approaches. A very recent work of Fripp et al. [10] proposes automatic segmentation of bone in order to extract the bone-cartilage interfaces (BCI) with promising results. However, similar to [7] we believe that current automatic approaches cannot achieve the high accuracy and precision needed in the clinical application. In most cases, interactive refinement is needed. Still, a good automatically achieved initialization could improve the daily work of radiologists, immensely. Therefore, we developed a novel approach for automatic segmentation of cartilage using a statistical atlas and efficient primal/dual linear programming. Our results provide a very good initialization for subsequent interaction steps and may even provide good enough segmentation results for certain applications.

The remainder of this paper is organized as follows; In Section 2 we present the construction of the probabilistic atlas, while in Section 3 we derive the formulation of the atlas-matching problem in a discrete setting. In Section 4 we briefly introduce the efficient optimization method based on primal/dual linear programming. Our results are presented in Section 5 and compared to the related methods. The last Section concludes our paper.

\section{Probabilistic Atlas Construction}

Let us assume that $n$ cartilage registered volumes are available $\mathcal{V}=\left\{\mathcal{V}_{1}, \mathcal{V}_{1}, \ldots, \mathcal{V}_{n}\right\}$. The task of atlas construction refers to the extraction of a model that combines the intensities of the training set to an average volume, a rather simplistic dimensionality reduction. On the other hand, a statistical atlas might be able to capture the variations of the training set, and often consists of

- $\mathcal{V}_{\mathcal{M}}: \Omega \rightarrow \mathcal{R}^{+}$that is an optimal representative volume - according to some criterion - derived from the training set,

- $\sigma_{\mathscr{M}}: \Omega \rightarrow \mathcal{R}^{+}$that is a variance map, determined according to the agreement between the atlas and the training set,

- $p_{\mathbf{x}}(i)$ : a $p d f$ defined at each voxel $\mathbf{x}$ which can be for instance a Gaussian density, $p_{\mathbf{x}}(i)=\frac{1}{\sqrt{2 \pi} \sigma_{\mathcal{M}}(\mathbf{x})} e^{-\frac{\left(i-\mathcal{V}_{\mathcal{M}}(\mathbf{x})\right)^{2}}{2 \sigma_{\mathscr{M}}(\mathbf{x})^{2}}}$.

with $\Omega$ being the volume domain. Towards the construction of such a probabilistic atlas representation [11] one can consider solving the inference problem at the voxel level $(\mathbf{x})$. Given a set of values $\left[\mathcal{V}_{1}(\mathbf{x}), \ldots, \mathcal{V}_{n}(\mathbf{x})\right]$, recover a distribution $\left(p_{\mathbf{x}}(i)\right)$ that has an optimal support from the data. The maximum posterior of this distribution along the training samples (assuming independences between voxels and using the [-log] of the density) is equivalent to minimizing

$$
E\left(\mathcal{V}_{\mathcal{M}}, \sigma_{\mathcal{M}}\right)=\iiint_{\Omega} \sum_{i=1}^{n}\left[\log \left(\sigma_{\mathcal{M}}^{2}(\mathbf{x})\right)+\frac{\left(\mathcal{V}_{i}(\mathbf{x})-\mathcal{V}_{\mathcal{M}}(\mathbf{x})\right)^{2}}{2 \sigma_{\mathcal{M}}^{2}(\mathbf{x})}\right] d \Omega
$$

However, volumes correspond to a collection of organs which exhibit certain spatial and intra-subject smoothness properties, therefore one can expect a smooth probabilistic 
atlas preserving contours/boundaries between organs. We can introduce such abstract constraints using regularization terms [12], which penalize the spatial derivatives of the field to be recovered:

$$
\begin{gathered}
E\left(\mathcal{V}_{\mathcal{M}}, \sigma_{\mathcal{M}}\right)=\alpha \iiint_{\Omega} \sum_{i=1}^{n}\left[\log \left(\sigma_{\mathcal{M}}^{2}(\mathbf{x})\right)+\frac{\left(\mathcal{V}_{i}(\mathbf{x})-\mathcal{V}_{\mathcal{M}}(\mathbf{x}(s))^{2}\right.}{2 \sigma_{\mathcal{M}}^{2}(\mathbf{x})}\right] d \Omega \\
\quad+\iiint_{\Omega} \psi\left(\nabla \sigma_{\mathcal{M}}(\mathbf{x})\right) d \Omega+\iiint_{\Omega} g\left(\left|\nabla \mathcal{V}_{\mathcal{M}}(\mathbf{x})\right|\right) \psi\left(\nabla \mathcal{V}_{\mathcal{M}}(\mathbf{x})\right) d \Omega
\end{gathered}
$$

where $\alpha$ is a constant that balances the data-fidelity and the smoothness term, $\psi$ (the quadratic form is considered in the scope of this paper) is a regularization function, and $g(|\nabla \mathcal{V}|)=\frac{1}{1+|\nabla \mathcal{V}|^{a}}$ a monotonically decreasing function which overwrites the smoothness constraint when a consensus for being at an edge is observed. The calculus of variations and a gradient descent method can now be used to recover the solution for the prior model $\left(\mathcal{V}_{\mathcal{M}}, \sigma_{\mathcal{M}}\right)$. One can initialize the process using one of the training volumes and constant variances. Furthermore, one can only consider a sub-domain of the volume domain which refers to a narrow-band zone from the cartilage since this is the component of interest.

\section{Cartilage Segmentation}

Let us now consider a new volume $\mathcal{V}:[1, X] \times[1, Y] \times[1, Z]$ to be segmented. We can reformulate segmentation as finding the region of interest in this volume, which best matches the atlas. In general, the two volumes are related with a non linear transformation $\mathcal{T}$, that minimizes

$$
E(\mathcal{T})=\iiint_{\Omega} \rho\left(\mathcal{V}(\mathcal{T}(\mathbf{x})), \mathcal{V}_{\mathcal{M}}(\mathbf{x}), \sigma_{\mathcal{M}}(\mathbf{x})\right) d \mathbf{x}=\iiint_{\Omega} \rho_{\mathcal{M}}(\mathcal{V}(\mathcal{T}(\mathbf{x}))) d \mathbf{x}
$$

where $\rho_{\mathcal{M}}$ is a distance metric used to determine the most meaningful correspondence between the atlas and the image domain $\Omega$. The distance metric in our approach could be explicitly defined using the probabilistic nature of the atlas, since $\mathcal{V}_{\mathcal{M}}(\mathbf{x})$ is assumed to be sample drawn from the density $p_{\mathbf{x}}()$, therefore one can expect that the optimal segmentation will project the voxel $\mathbf{x}$ of the atlas to image intensity $\mathcal{V}(\mathcal{T}(\mathbf{x}))$ that refers to the maximum of $p_{\mathbf{x}}()$ leading to the minimization of

$$
\rho_{\mathcal{M}}(\mathcal{V}(\mathcal{T}(\mathbf{x})))=\left[\log \left(\sigma_{\mathcal{M}}^{2}(\mathbf{x})\right)+\frac{\left(\mathcal{V}(\mathcal{T}(\mathbf{x}))-\mathcal{V}_{\mathcal{M}}(\mathbf{x})\right)^{2}}{2 \sigma_{\mathcal{M}}^{2}(\mathbf{x})}\right]
$$

Since we are interested in local registration (assuming a global pre-registration exists), let us introduce a deformation grid $\mathcal{G}:[1, K] \times[1, L] \times[1, M]$ (usually $K \ll X$, $L \ll Y$, and $M \ll Z$ ) super-imposed to the atlas. The central idea of our approach is to deform the grid (with a $3 \mathrm{D}$ displacement vector $\mathbf{d}_{p}$ for each control point) such that the structures in the atlas and the image to be segmented are perfectly aligned. The transformation of a voxel $\mathbf{x}$ can be expressed using a linear or non-linear combination of the grid points, or

$$
\mathcal{T}(\mathbf{x})=\mathbf{x}+\mathcal{D}(\mathbf{x}), \quad \mathcal{D}(\mathbf{x})=\sum_{p \in \mathcal{G}} \eta(|\mathbf{x}-\mathbf{p}|) \mathbf{d}_{p}
$$


where $\eta(\cdot)$ is the weighting function measuring the contribution of the control point $p$ to the displacement field $\mathcal{D}$. The position of point $p$ is denoted as $\mathbf{p}$. In such a theoretical setting without loss of generality we consider Free Form Deformations (FFD) based on cubic B-splines as a transformation model which have been often considered for image registration [13].

By defining the atlas-matching problem based on such a deformation model we can now reformulate the criterion earlier introduced,

$$
E_{\text {data }}(\mathcal{T})=\sum_{p \in \mathcal{G}} \iiint_{\Omega} \eta^{-1}(|\mathbf{x}-\mathbf{p}|) \rho_{\mathcal{M}}(\mathcal{V}(\mathcal{T}(\mathbf{x}))) d \mathbf{x}
$$

where $\eta^{-1}(\cdot)$ is the inverse projection for the contribution to the objective of the image point $\mathbf{x}$ according to the influence of the control point $p$.

Such a term will guarantee intensity correspondence between the two images. Hence, this term is also called the data term. The transformation due to the interpolation inherits some implicit smoothness properties. However, in order to avoid folding of the deformation grid, one can consider a smoothness term on the grid domain, or

$$
E_{\text {smooth }}(\mathcal{T})=\sum_{p \in \mathcal{G}} \phi\left(\left|\nabla_{\mathcal{G}} \mathbf{d}_{p}\right|\right)
$$

with $\phi$ being a smoothness penalty function for instance penalizing the first derivatives of the grid deformation. The complete term associated with the registration problem is then defined as the sum of the data and smoothness term. The most common way to obtain the transformation parameters is through the use of a gradient-descent method in an iterative approach which due to the non-convexity of the cost function could produce sub-optimal results. One way to overcome this constraint is through the use of more efficient optimization techniques, like combinatorial programming [14].

Let us now consider a discrete set of labels $\mathcal{L}=\left\{u^{1}, \ldots, u^{i}\right\}$ corresponding to a quantized version of the deformation space $\times=\left\{d^{1}, \ldots, d^{i}\right\}$. A label assignment $u_{p}$ to a grid node $p$ is associated with displacing the node by the corresponding vector $d^{u_{p}}$. The image transformation associated with a certain discrete labeling $u$ becomes

$$
\mathcal{D}(\mathbf{x})=\sum_{p \in \mathcal{G}} \eta(|\mathbf{x}-\mathbf{p}|) d^{u_{p}}
$$

One can reformulate the registration as a discrete optimization problem, that is assign individual labels $u_{p}$ to the grid nodes such that

$$
E_{\text {data }}(u)=\sum_{p \in \mathcal{G}} \iiint_{\Omega} \eta^{-1}(|\mathbf{x}-\mathbf{p}|) \rho_{\mathcal{M}}(\mathcal{V}(\mathcal{T}(\mathbf{x}))) d \mathbf{x} \approx \sum_{p \in \mathcal{G}} V_{p}\left(u_{p}\right)
$$

where $V_{p}(\cdot)$ represents a local similarity metric. In this setting, the singleton potential functions $V_{p}(\cdot)$ are not independent, thus the defined data term can only be approximated. Hence, we pre-compute the $|\mathcal{L}| \times|\mathcal{G}|$ data term look-up table for the atlas and a given image by simple shift operators. The entry for node $p$ and labels $u_{p}$ is determined by

$$
V_{p}\left(u_{p}\right)=\iiint_{\Omega_{p}} \rho_{\mathscr{M}}(\mathcal{V}(\mathcal{T}(\mathbf{x}))) d \mathbf{x}
$$


We determine the metric directly from the image patch $\Omega_{p}$ centered at node $p$. Thus, the weighting function $\eta^{-1}(\cdot)$ can be neglected.

In order to define the smoothness in the label domain one can express distances between the deformation vectors using difference between labels if a ranking has been considered within the definition of the label set, or

$$
E_{\text {smooth }}(u)=\sum_{p, q \in \mathcal{E}(p)} V_{p q}\left(u_{p}, u_{q}\right), \quad V_{p q}\left(u_{p}, u_{q}\right)=\min \left(\left|d^{u_{p}}-d^{u_{q}}\right|, T\right)
$$

where $\mathcal{E}$ represents the neighborhood system associated with the deformation grid $\mathcal{G}$. For the distance $V_{p q}(\cdot, \cdot)$ we consider a simple piecewise smoothness truncated term based on the euclidean geometric distances between the deformations corresponding to the assigned labels with $T$ being the maximum penalty. Such a smoothness term together with the data term allows to convert the problem of image registration into the form of a Markov Random Field (MRF) in a discrete domain, or

$$
E_{\text {total }}(u)=\sum_{p \in \mathcal{G}} V_{p}\left(u_{p}\right)+\sum_{p, q \in \mathcal{E}(p)} V_{p q}\left(u_{p}, u_{q}\right) .
$$

\section{Linear Programming}

For optimizing the above discrete Markov Random Field, we will make use of a recently proposed method, we call Fast-PD [15]. This is an optimization technique, which builds upon principles drawn from the duality theory of linear programming in order to efficiently derive almost optimal solutions for a very wide class of NP-hard MRFs. Instead of working directly with the discrete MRF optimization problem above, Fast-PD first reformulates that problem as an integer linear programming problem (the primal problem) and also takes the dual of the corresponding LP relaxation. Given these 2 problems, i.e. the primal and the dual, Fast-PD then generates a sequence of integral feasible primal solutions, as well as a sequence of dual feasible solutions. These two sequences of solutions make local improvements to each other until the primal-dual gap (i.e. the gap between the objective function of the primal and the objective function of the dual) becomes small enough. Once this happens, the last generated primal solution is guaranteed to be an approximately optimal solution, i.e. within a certain distance from the optimum (in fact, this distance can be shown to be smaller than the achieved primal-dual gap). This is exactly what the next theorem, also known as the primal-dual principle, states.

Primal-Dual Principle 1 (Primal-Dual principle). Consider the following pair of primal and dual linear programs:

$$
\begin{aligned}
& \text { PRIMAL: } \min \mathbf{c}^{T} \mathbf{x} \quad \text { DUAL: } \max \mathbf{b}^{T} \mathbf{y} \\
& \text { s.t. } \mathbf{A x}=\mathbf{b}, \mathbf{x} \geq \mathbf{0} \quad \text { s.t. } \quad \mathbf{A}^{T} \mathbf{y} \leq \mathbf{c}
\end{aligned}
$$

and let $\mathbf{x}, \mathbf{y}$ be integral-primal and dual feasible solutions, having a primal-dual gap less than $f$, i.e.:

$$
\mathbf{c}^{\mathbf{T}} \mathbf{x} \leq f \cdot \mathbf{b}^{\mathbf{T}} \mathbf{y} .
$$

Then $\mathbf{x}$ is guaranteed to be an $f$-approximation to the optimal integral solution $x^{*}$, i.e., $\mathbf{c}^{\mathbf{T}} \mathbf{x}^{*} \leq \mathbf{c}^{\mathbf{T}} \mathbf{x} \leq f \cdot \mathbf{c}^{\mathbf{T}} \mathbf{x}^{*}$ 


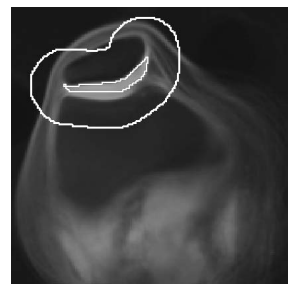

(a)

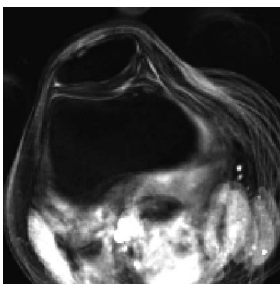

(b)

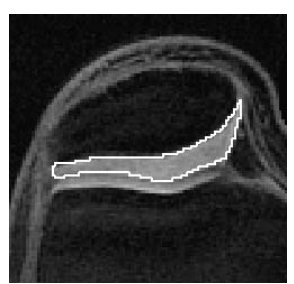

(c)

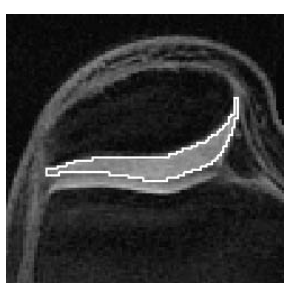

(d)

Fig. 1. (a) $\mathcal{V}_{\mathcal{M}}$ and (b) $\sigma_{\mathcal{M}}$ of the statistical atlas. In (a) the region of interest for the local registration and the atlas segmentation are overlaid. (c) Automatic segmentation compared to (d) ground truth. For this example the DSC of the segmented volume is 0.90 and the sensitivity is $93.28 \%$. The lower interface between cartilage and surrounding soft-tissue is well preserved.

Fast-PD is a very general MRF optimization method, which can handle a very wide class of MRFs. Essentially, it only requires that the pairwise potential function is nonnegative (i.e., $V_{p q}(\cdot, \cdot) \geq 0$ ). Furthermore, as already mentioned, it can guarantee that the generated solution is always within a worst-case bound from the optimum. In fact, besides this worst-case bound, it can also provide per-instance approximation bounds, which prove to be much tighter, i.e. very close to 1 , in practice. It thus allows the global optimum to be found up to a user/application bound. Finally, it provides great computational efficiency, since it is typically 3-9 times faster than any other MRF optimization technique with guaranteed optimality properties.

\section{Experiments}

We evaluated our segmentation method on 56 T2-weighted MRI data sets of probands each with a resolution of $256 \times 256 \times 20$ and a voxel size of $0.625 \times 0.625 \times 3.0$ millimeters. The patella cartilage is manually segmented by clinical experts in a slice-by-slice fashion in all volumes. We are using 28 data sets for the atlas construction and segment automatically the remaining 28 data sets using the atlas-matching approach described above. We perform a (standard) global pre-registration with 9 degrees of freedom (translation, rotation, and scaling) using available methods1. In such a pre-registration we only consider the $\mathcal{V}_{\mathcal{M}}$ of the atlas. The global registration provides a sufficient initialization for our subsequent non-rigid atlas-matching. All experiments are done on an Intel Centrino $2.16 \mathrm{GHz}$ machine. A full registration of two images including the global pre-registration takes about 45 seconds where our non-rigid atlas-matching takes less than 20 seconds. The matching is done in a pyramidal fashion with two resolution levels. The quantized displacement space is sampled with five steps in the six main 3D directions with a step size of $0.8 \mathrm{~mm}$ for the coarser resolution level and $0.25 \mathrm{~mm}$ on the full volume resolution. On each level we perform ten iterations which are sufficient to let the matching converge. The control point spacing of the deformation grid is set to $10 \mathrm{~mm}$ for the coarser level and $5 \mathrm{~mm}$ for the finer pyramid level.

\footnotetext{
${ }^{1}$ National Library of Medicine Insight Segmentation and Registration Toolkit.
} 
Table 1. Comparison of different methods for cartilage segmentation. In the upper part the results of semi-automatic methods are shown. The lower part shows fully automatic methods.

\begin{tabular}{|l|c|c|c|c|l|}
\hline Semi-automatic & DSC Mean $($ Std $)$ & Sensitivity & Specificity & Interaction & Cartilage \\
\hline Grau et al. [8] & $0.90( \pm 0.01)$ & $90.03 \%$ & $99.87 \%$ & $5-10$ min & Tibia, Femur, Patella \\
Dam et al. [7] & $0.92( \pm n / a)$ & $93.00 \%$ & $99.99 \%$ & max 10 min & Tibia, Femur \\
\hline Automatic & DSC Mean (Std) & Sensitivity & Specificity & Interaction & Cartilage \\
\hline Cheong et al. [17 & $0.64( \pm 0.15)$ & $74.00 \%$ & $\mathrm{n} / \mathrm{a}$ & 0 & Medial Tibia \\
Cheong et al. [17 & $0.72( \pm 0.09)$ & $79.00 \%$ & $\mathrm{n} / \mathrm{a}$ & 0 & Lateral Tibia \\
Folkesson et al. [6] & $0.80( \pm 0.03)$ & $90.01 \%$ & $99.80 \%$ & 0 & Tibia, Femur \\
Our Approach & $0.84( \pm 0.06)$ & $94.06 \%$ & $99.92 \%$ & 0 & Patella \\
\hline
\end{tabular}

In order to evaluate the segmentation results we compute several common measurements [16], namely the Dice similarity coefficient (DSC), the sensitivity, the specificity, and the average surface distance (ASD) from the manual segmentations. The ASD is computed in millimeters from an anisotropic 3D Euclidean distance transform of the automatic and manual segmentation surfaces and the overlay of the respective surface. We achieve an ASD of $0.49( \pm 0.23) \mathrm{mm}$ which is below the voxel size. In Table 5 we compare our results to methods reported in the literature.

However, due to the variability in the MRI sequences, volume resolution, and target anatomy such a direct comparison can only give an idea about the performance of the different methods. Compared to the semi-automatic methods [78] we get a worse DSC, but a slightly better sensitivity and specificity. Assuming that most of the errors occur at the boundaries, small object such as patella cartilage are penalized and get lower DSC score than larger objects such

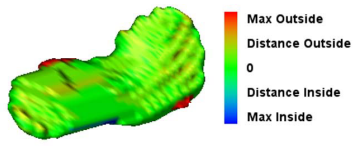

Fig. 2. Color-encoded visualization of the average surface distance for the example shown in Fig. 4 (c)/(d) as the tibial or femoral cartilage. Still, our approach obtains better results than the automatic classification scheme proposed in [6]. From [17] we consider only the results of the proposed Patch-based Active Appearance Model, since it performs best in their evaluation of different automatic model-based approaches. Fripp et al. [10] report a median DSC for the bone-cartilage interface (BCI) of 0.93. However, the extraction of BCI does not provide closed volumes and thus is not directly comparable to our method.

\section{Conclusion}

In this paper we have proposed a novel approach for fully automatic segmentation of knee cartilage using a statistical atlas and efficient primal/dual linear programming. We could achieve segmentation results for proband data with a mean overlap ratio of 0.84 , a sensitivity and specificity of $94.06 \%$ and $99.92 \%$ compared to manual expert segmentation. Such an automatic approach could provide significant initialization improvements in applications with high accuracy constraints where interactive refinements are unavoidable. In less constrained applications such as rough classification tasks our method could even provide the final results. We will evaluate the robustness of our approach on patient data including higher variations in shape appearance. In future work also more complex priors including shape models will be investigated and the method will also be extended to other anatomy. 


\section{References}

1. Yelin, E.: Cost of musculoskeletal diseases: Impact of work disability and functional. J. Rheumatol. 68(Suppl.), 8-11 (2003)

2. Eckstein, F., Cicuttini, F., Raynauld, J., Waterton, J., Peterfly, C.: Magnetic resonance imaging (mri) of cartilage in knee osteoarthritis (oa): morphological assesment. Osteoarthr. Cartil. 14, 46-75 (2006)

3. Kauffmann, C., Gravel, P., Godbout, B., Gravel, A., Beaudoin, G., Raynauld, J.P., MartelPelletier, J., Pelletier, J.P., de Guise, J.: Computer-aided method for quantification of cartilage thickness and volume changes using mri: validation study using a synthetic model. IEEE Biomedical Engineering 50(8), 978-988 (2003)

4. Tamez-Pena, J., Barbu-McInnis, M., Totterman, S.: Unsupervised definition of the tibiafemoral joint regions of the human knee and its applications to cartilage analysis. In: SPIE Medical Imaging, San Diego (2006)

5. Tang, J., Millington, S., Acton, S., Crandall, J., Hurwitz, S.: Surface extraction and thickness measurement of the articular cartilage from $\mathrm{mr}$ images using directional gradient vector flow snakes. IEEE Biomedical Engineering 53(5), 896-907 (2006)

6. Folkesson, J., Dam, E., Olsen, O.F., Pettersen, P., Christiansen, C.: Automatic segmentation of the articular cartilage in knee mri using a hierarchical multi-class classification scheme. In: Duncan, J.S., Gerig, G. (eds.) MICCAI 2005. LNCS, vol. 3749, Springer, Heidelberg (2005)

7. Dam, E., Folkesson, J., Pettersen, P., Christiansen, C.: Semi-automatic knee cartilage segmentation. In: SPIE Medical Imaging, San Diego (2006)

8. Grau, V., Mewes, A., Alcaniz, M., Kikinis, R., Warfield, S.: Improved watershed transform for medical image segmentation using prior information. IEEE Medical Imaging 23(4), 447458 (2004)

9. Cheong, J., Suter, D., Cicuttini, F.: Development of semi-automatic segmentation methods for measuring tibial cartilage volume. In: Digital Image Computing: Technqiues and Applications, DICTA 2005. Proceedings, pp. 307-314 (2005)

10. Fripp, J., Crozier, S., Warfield, S., Ourselin, S.: Automatic segmentation of the bone and extraction of the bone-cartilage interface from magnetic resonance images of the knee. Physics in Medicine and Biology (2007)

11. Rousson, M., Paragios, N.: Prior knowledge, level set representations and visual grouping. International Journal of Computer Vision (in press)

12. Tikhonov, A.: Ill-posed problems in natural sciences, Coronet (1992)

13. Schnabel, J.A., Rueckert, D., Quist, M., Blackall, J.M., Castellano-Smith, A.D., Hartkens, T., Penney, G.P., Hall, W.A., Liu, H., Truwit, C.L., Gerritsen, F.A., Hill, D.L.G., Hawkes, D.J.: A generic framework for non-rigid registration based on non-uniform multi-level free-form deformations. In: Niessen, W.J., Viergever, M.A. (eds.) MICCAI 2001. LNCS, vol. 2208, Springer, Heidelberg (2001)

14. Glocker, B., Komodakis, N., Paragios, N., Tziritas, G., Navab, N.: Inter and intra-modal deformable registration: Continuous deformations meet efficient optimal linear programming. In: Information Processing in Medical Imaging, Kerkrade, Netherlands (2007)

15. Komodakis, N., Tziritas, G., Paragios, N.: Fast, approximately optimal solutions for single and dynamic mrfs. In: Computer Vision and Pattern Recognition (2007)

16. Gerig, G., Jomier, M., Chakos, M.: Valmet: A new validation tool for assessing and improving 3d object segmentations. In: Niessen, W.J., Viergever, M.A. (eds.) MICCAI 2001. LNCS, vol. 2208, Springer, Heidelberg (2001)

17. Cheong, J., Faggian, N., Langs, G., Suter, D., Cicuttini, F.: A comparison of model-based methods for knee cartilage segmentation. In: Computer Vision Theory and Applications (2007) 